\title{
Results from the Pierre Auger Observatory
}

\author{
Daniel V. Camin* \\ Dipartimento di Fisica and Sezione INFN Milano, Italy \\ E-mail: Daniel.Victor.Camin@mi.infn.it
}

\section{Cinzia De Donato}

Dipartimento di Fisica and Sezione INFN Milano, Italy

E-mail: cinzia.dedonato@mi.infn.it

\section{for the Auger Collaboration}

The Pierre Auger Observatory has been designed to substantially increase the statistics of cosmic rays from $10^{18} \mathrm{eV}$ up to the highest energies. The Observatory comprises a Surface Detector which consists of an array of 1600 water Cherenkov tanks spaced each other by $1.5 \mathrm{~km}$ over an area of $\sim 3000 \mathrm{~km}^{2}$, in a region known as Pampa Amarilla in the Northern Patagonia, Argentina. The Surface Detector is sensitive to the shower front that sequentially triggers the tanks interested by the event. At the periphery of the site there are four buildings, the Optical Stations, each one including six large-aperture telescopes optimized for maximum signal-to-noise ratio in the UV range of the spectrum. The UV light is originated by the interaction of the charged particles in the shower with the Nitrogen atoms in the atmosphere. The Fluorescence Detector comprises all four Optical Stations, of which the last one at Loma Amarilla is scheduled to be completed within 2006. The events could be seen by a single optical station, by two or more optical stations, and by both the Surface Detector and the Fluorescente Detector. The latter, hybrid, modality ensures an accurate determination of the shower's energy by combining the data obtained with both techniques. This is an outstanding feature of the Pierre Auger Observatory. An overview of the Observatory and a selection of the most interesting and relevant events will be reported.

International Europhysics Conference on High Energy Physics

July 21st - 27th 2005

Lisboa, Portugal

\footnotetext{
* Speaker.
} 


\section{Introduction}

The Pierre Auger Observatory (PAO) has been conceived to study the Ultra High Energy Cosmic Rays (UHERC) with energy above $10^{18} \mathrm{eV}$ with full efficiency above $10^{19} \mathrm{eV}$. The aim of the Pierre Auger Project is to give an answer to the origin of such energetic particles by measuring the energy spectrum, the mass composition and the arrival direction of the shower.

In order to increase the collecting power of the previous experiments with a full sky coverage, the Auger Project foresees two sites (one in each hemisphere), each one with an aperture of $7850 \mathrm{Km}^{2} \mathrm{sr}$. Each site consists of a Surface Detector (SD), a ground array of 1600 waterCherenkov tanks deployed over a $3000 \mathrm{Km}^{2}$ area and spaced each other by $1.5 \mathrm{~km}$; The Fluorescence Detector (FD) is constituted by four optical stations at the periphery of the SD array, looking inwards, each one composed of 6 fluorescence telescopes. The LIDAR and optical calibration systems complete the Observatory. The SD allows an accurate sampling of the particle density distribution at ground, i.e. the lateral density distribution.The FD measures the longitudinal development of the cosmic ray shower in the atmosphere above the earth. This is done by detecting the fluorescence photons emitted isotropically by the nitrogen molecules excited by the shower's secondary particles propagating in the atmosphere. From the lateral density distribution and the longitudinal profile, the parameters of the shower can be inferred independently.

To achieve an unprecedent statistical precision, the project combines for the first time the two traditional techniques in a unique hybrid detector. The combined use of the information obtained with the two detectors allows the reduction of the systematic errors thanks to the cross-calibration of the detectors.

The southern site is under construction at Malargue (Mendoza, Argentina) at an altitude of $1400 \mathrm{~m}$ and will be completed within the end of 2006. Since January 2004 the observatory is regularly acquiring data. The array status by June 2005 is shown in Fig. 1 with 740 tanks and 3 optical station fully operational; the last optical station at Loma Amarilla is scheduled to be completed within 2006. In the same figure the cores of the reconstructed hybrid events are also shown (§4.1).

A detailed description of the project is given in [1].

\section{The Surface Detector}

The SD consists of 1600 water-Cherenkov tanks of $10 \mathrm{~m}^{2}$ cross section and about $1.5 \mathrm{~m}$ high, filled with pure water. Three 9" Photonis XP1805 photomultiplier tubes (PMT) are used to collect the Cherenkov light emitted by the shower particles traversing the tanks, which is approximately proportional to the energy deposited. The signal is extracted from the anode and the last dynode and it is digitized at $40 \mathrm{MHz}$ by a $10 \mathrm{bit}$ FADC. To achieve a large dynamic range (15 bit extending from a few to $10^{5}$ photoelectrons) the dynode signal is amplified by a factor 32 . The timing between the tanks is obtained by a GPS Motorola unit that ensures a resolution of $8 n s$ [2]. The power for each tank $(\sim 10 \mathrm{~W})$ is supplied by two solar panels. The comunication to the Central Data Acquisition System (CDAS) is done via a wireless comunication system.

The detector calibration is inferred from background muons [3,4]. The measurement of the muon charge spectrum allows the deduction of the Vertical Equivalent Muon (VEM) value from which the calibration is inferred for the whole dynamic range. Furthermore, the area/peak of the muon charge 
spectrum can be used to monitor the stability and uniformity of the array during the whole lifetime of the experiment [5]. On the other hand, the cross-calibration between the anode and dynode channels is performed using small shower signals in the overlap region of the two channels.

\section{The Fluorescence Detector}

Each FD station consists of a building with six bays, each one containing a telescope sensitive to the UV photons with a field of view of $30^{\circ}$ in azimuth and $28.6^{\circ}$ in elevation.

Each telescope incorporates Schmidt optics to reduce the optical aberrations and to increse the signal-to-noise ratio, while the aperture is defined by the circular diaphragm placed at the center of curvature of the spherical mirror.

The focal surface of the telescopes consists of a camera in which there are installed 440 photomultiplier tubes Photonis XP3062 (PMT) arranged in a $20 \times 22$ array. An UV trasmitting filter (MUG-6 glass sheets), matched with the fluorescence spectrum $(300-400 \mathrm{~nm})$ and installed in the $2.2 \mathrm{~m}$ diameter aperture, improves the signal-to-noise ratio. The light entering the telescope diaphragm is collected by a spherical mirror with an area of $3.5 \mathrm{~m} \times 3.5 \mathrm{~m}$, a radius of curvature of $3.4 \mathrm{~m}$ and $1.743 \mathrm{~m}$ focal distance. A corrector ring installed in the periphery of the aperture, complete the optical components.

Each PMT is equipped with a Head Electronics unit (HE) which provides active bias. The active network improves linearity over the whole dynamic range and reduces the $\mathrm{HV}$ cost by $50 \%$, compared to a standard passive base. The PMT signals are digitized in the Front-End Board (FEB) at a $10 \mathrm{MHz}$ sample rate with 12 bit resolution. The dynamic range is increased to 15 bit by sampling with a lower gain the signal sum of groups of 11 pixels in a column.

The internal timing resolution of the FD is $7 n s$ between pixels of the same camera and $20 n s$ between different telescopes [2]. The comunication to the Central Data Acquisition System (CDAS) is done via a wireless comunication system. The calibration procedures are fully described in [1].

\section{The Hybrid detector}

The most innovative feature of the Pierre Auger Observatory is the combined use of the two traditional techniques used separately in previous experiments: the SD and FD that allow the hybrid reconstruction of events recorded during the dark data taking period. As the duty cycle of the FD is only $10 \%$, most of the statistics arrives from the SD events. On the other hand, the detection in coincidence of a shower by the FD and SD allows the cross-calibration and consequentely the reduction of the systematic errors in the SD reconstruction. In this section the first results from the analysis of the hybrid events acquired by the observatory will be reported.

\subsection{Data acquired}

The data analyzed have been acquired from 1 January 2004 to 5 June 2005. The cumulative exposure adds up to $1750 \mathrm{~km}^{2}$ sr $y r, 7 \%$ more than the AGASA total exposure ${ }^{1}$. The position of

\footnotetext{
${ }^{1}$ During the exposition period, the mean dimension of the observatory was only $\sim 22 \%$ of the total area of the Southern observatory.
} 


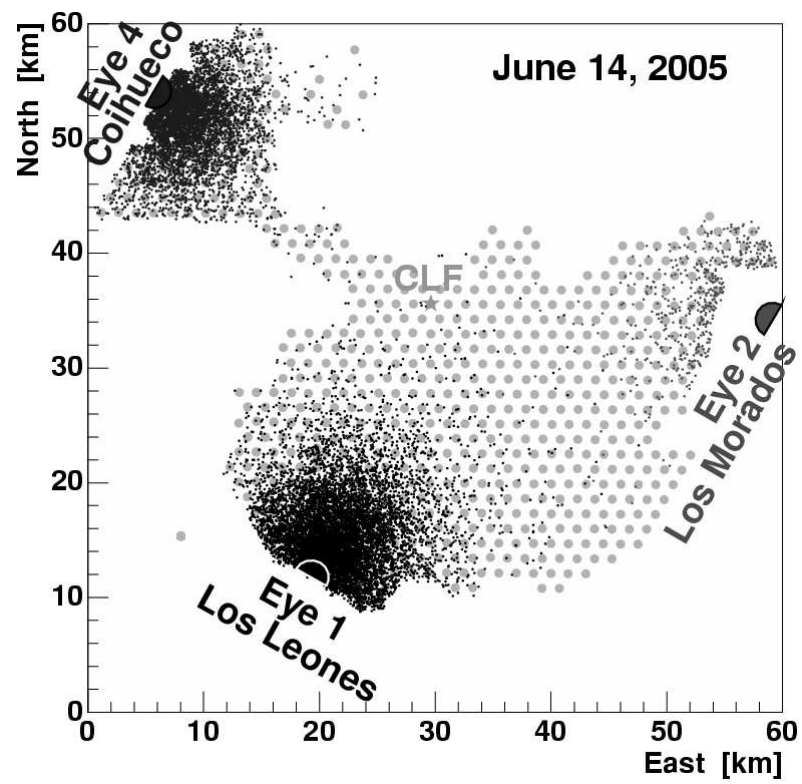

Figure 1: Hybrid events collected in the period January 2004 - June 2005, for a total number of 16090 showers. The position of the core of each shower is shown. The 740 tanks (light gray spot) and the three optical station operational are shown.

the core of the hybrid reconstructed events are shown in Fig.1. The mean rate at June 2005 was 50 events/night per optical station for a total number of 16090 hybrid events.

A selection of the best hybrid events has been done for further analysis, giving 3525 hybrid events with $E>3 \mathrm{EeV}$ and $\theta_{\text {zenith }}<60^{\circ}$. The event acceptance criteria used and the exposure calculation are described in $[6,7]$.

\subsection{Energy determination: hybrid resolution and spectrum}

The SD event reconstruction procedure is fully described in $[8,1]$, as well as the FD reconstruction procedure described in $[9,1]$. The signals measured by each SD station $\left(S_{\text {meas }}\right)$ are compared with those expected (S) from the lateral distribution function (LDF). The experimental determination of the LDF is described in [10].

For the geometry of the SD and for the energy range investigated, the water-Cherenkov signal at a distance of $1000 \mathrm{~m}$ from the shower core, S(1000), is the best energy estimator, indipendently of the composition of the primary. As the attenuation of $S(1000)$ depends on the slant dephts and consequently on the zenith angle of the axis, this value is converted to the new parameter $S_{38}$, the signal that would have been produced from the analyzed shower at a distance of $1000 \mathrm{~m}$ if the shower had arrived at a zenith angle $\theta=38^{\circ}$ [10].

This parameter is well correlated with the FD energy measurements in hybrid events, reconstructed independently by the FD and SD [11]. The FD reconstructed energy vs the parameter $S_{38}$ for hybrid events is shown in Fig.2(a).

The fitted line provides an empirical rule for assigning energies based on the parameter $S_{38}$ (in VEM):

$$
E=0.16 \times S_{38}^{1.06}(E e V)
$$




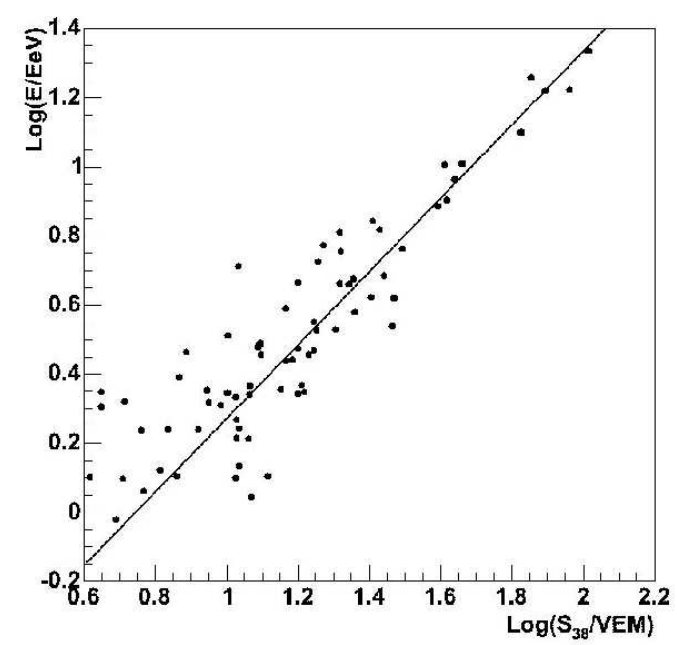

(a) FD energy vs ground parameter $S_{38}$.

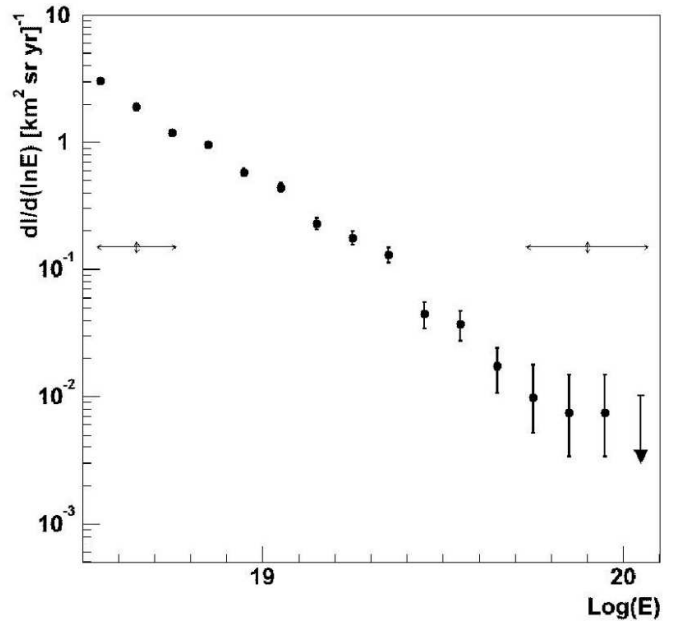

(b) First estimate of the primary cosmic ray energy spectrum above $3 \mathrm{EeV}$.

Figure 2: Analysis of the selected hybrid events with $E>3 \mathrm{EeV}$ and $\theta_{\text {zenith }}<60^{\circ}$ [11]. For further details see the text.

The total systematic error in the conversion of $S_{38}$ to energy is due to different contributions. The main contribution comes from the energy resolution of the FD reconstruction. Actually the total systematic uncertainty in the FD energy measurements is estimated to be $25 \%$, mainly given by a $15 \%$ uncertainty in the fluorescence yield and $12 \%$ uncertainty in the absolute calibration [9].

Another important factor is the uncertainty in the determination of $\mathrm{S}(1000)$; the statistical uncertainties for a primary energy $E=5 \cdot 10^{18} \mathrm{eV}$ are of the order of $10 \%$, while the systematic uncertainties due to the assumed LDF form are $<4 \%$ [8]. Besides, the accuracy of the fit shown in Fig.2(a) is limited by the available statistics. Combining all these contributions, the total systematic error in the conversion of $S_{38}$ to energy grows from $30 \%$ at $3 \mathrm{EeV}$ to $50 \%$ at $100 \mathrm{EeV}$ [11].

From the data analyzed, a first estimate of the primary cosmic ray energy spectrum above $3 \mathrm{EeV}$ can be derived. In Fig.2(b) it is plotted the differential intensity $\frac{d I}{d \ln E}$ for the selected 3525 hybrid events (\$4.1). The error bars on points indicate the statistical errors (95\% CL upper limit), given by the Poisson uncertainty in the number of measured shower, while the systematic errors in the energy determination are shown by horizontal arrows and grows with energy.

\section{Conclusions}

The completion of the array is at 75\% for the FD and 50\% for the SD. The Southern Observatory is scheduled to be completed by the end of 2006. From January 2004 the observatory began to acquire data regularly. At present a hybrid event rate of 50 events/night per optical station has been reached, for a total number of 16090 hybrid events. The actual cumulative exposure adds up to $1750 \mathrm{~km}^{2} \mathrm{sr} y \mathrm{r}, 7 \%$ more than the AGASA total exposure. The analysis of the best data acquired 
has provided an empirical rule for the conversion of the ground parameter $S_{38}$ to energy, useful for the cross-calibration of the FD/SD detectors. The hybrid energy resolution achieved grows from $30 \%$ at $3 \mathrm{EeV}$ to $50 \%$ at $100 \mathrm{EeV}$, limited by the available statistics and by the systematic errors of the FD and SD reconstruction. An improvement of the hybrid recostruction is expected from the grow of the array, and consequently of the statistics. A first estimate of the cosmic ray spectrum above $3 \mathrm{EeV}$ has been done with the available statistics.

\section{References}

[1] J. Abraham, et al., Properties and performance of the prototype instrument for the Pierre Auger Observatory, Nuclear Instruments and Methods, A523 (2004), pp. 50-95.

[2] P. Allison et al., Pierre Auger Collaboration, Timing calibration and synchronization of surface and fluorescence detectors of the Pierre Auger Observatory, in proceedings of the 29th ICRC, Pune, India (2005).

[3] P. Allison et al., Pierre Auger Collaboration, Observing muon decays in water Cherenkov detectors at the Pierre Auger Observatory, in proceedings of the 29th ICRC, Pune, India (2005) [astro-ph/0509238].

[4] M. Aglietta et al., Pierre Auger Collaboration, Calibration of the surface array of the Pierre Auger Observatory, in proceedings of the 29th ICRC, Pune, India (2005).

[5] I. Allekotte et al., Pierre Auger Collaboration, Observation of long term stability of water tanks in the Pierre Auger surface detector, in proceedings of the 29th ICRC, Pune, India (2005).

[6] D. Allard et al., Pierre Auger Collaboration, Aperture calculation of the PAO surface detector, in proceedings of the 29th ICRC, Pune, India (2005) [astro-ph/ 0511104 ].

[7] D. Allard et al., Pierre Auger Collaboration, The trigger system of the PAO surface detector: operation, efficiency and stability, in proceedings of the 29th ICRC, Pune, India (2005) [astro-ph/0510320].

[8] Pierre Auger Collaboration, Statistical and systematic uncertainties in the event reconstruction and $S$ (1000) determination by the Pierre Auger surface detector, in proceedings of the 29th ICRC, Pune, India (2005) [astro-ph/ 0507029 ].

[9] Pierre Auger Collaboration, Performance of the fluorescence detectors of the Pierre Auger Observatory, in proceedings of the 29th ICRC, Pune, India (2005) [astro-ph / 0508389 ].

[10] D. Barnhill et al., Pierre Auger Collaboration, Measurement of the lateral distribution function of the UHECR air showers with the Auger Observatory, in proceedings of the 29th ICRC, Pune, India (2005) [astro-ph/0507590].

[11] Pierre Auger Collaboration, First Estimate of the primary cosmic ray spectrum above 3 EeV from the Pierre Auger Observatory, in proceedings of the 29th ICRC, Pune, India (2005)

[astro-ph/0507150]. 